\title{
Oncocitoma renal. Revisión de nuestra serie de 22 pacientes
}

\author{
Argüelles Salido E, Marcilla Plaza D*, Medina López R, Congregado Ruiz B, Campoy Martínez P, \\ Cayuela Domínguez A**.
}

Servicio de Urología. *Servicio de Anatomía Patológica. ** Unidad de Apoyo a la Investigación. Hospital Universitario Virgen del Rocío.

Actas Urol Esp 2006; 30 (6): 583-590

\section{RESUMEN}

ONCOCITOMA RENAL. REVISIÓN DE NUESTRA SERIE DE 22 PACIENTES.

Introducción: El oncocitoma renal es un tumor benigno cuya frecuencia de presentación oscila entre el 37\% de las masas renales sólidas. Muestra especiales características celulares y evolutivas, con posibilidad de metacronicidad, bilateralidad y multifocalidad, además de un difícil diagnóstico diferencial con neoplasias malignas.

Material y Método: Revisamos retrospectivamente en 428 tumores renales intervenidos desde Enero de 1986 hasta Abril de 05 la proporción de oncocitomas diagnosticados, analizando sus características anatomopatológicas y comportamiento clínico, comparándolas con las presentadas por los carcinomas de células renales. Observamos su posible bilateralidad, multifocalidad y asociación con otros tumores. Determinamos en cuántos casos los métodos diagnósticos de imagen permitieron sospechar la naturaleza tumoral benigna previamente a la intervención. Seguimiento medio y mediano 13 y 36,86 meses respectivamente (1-193).

Resultados: Hemos encontrado 24 oncocitomas (5,67\%), en 10 hombres y 12 mujeres con una edad media de 59 años (34-84). 12 de ellos en riñón izquierdo y 12 en derecho, además de una oncocitomatosis renal. Tamaño medio de 4,64 cm (1-12,5). En 17 pacientes el diagnóstico ha sido incidental. En los 5 restantes el síntoma de debut fue hematuria. Supervivencia específica 100\%. Se sospechó la naturaleza tumoral benigna o específicamente oncocitomatosa previamente a la cirugia en 22,73\% (5/22) y 9,9\% (2/22) respectivamente.

Conclusiones: En nuestra serie de tumores renales intervenidos el porcentaje de oncocitomas coincide con lo publicado en la literatura. Hemos encontrado dos casos de tumor sincrónico, pero ninguno metacrónico, bilateral ni metastásico. Todos han presentado un comportamiento benigno.

Palabras clave: Oncocitoma renal. Neoplasias renales. Tumor renal benigno.

\section{ABSTRACT}

RENAL ONCOCYTOMA. REVIEW OF OUR 22 PATIENTS

Summary: Renal oncocytoma (OR) is a benign tumor. It may represent up to 3-7\% of solid kidney masses, and shows specifics cellular and evolutive characteristics. Metacronicity, multifocality and bilateralism has been reported.

Materials and methods: Between 1986 and 2005, 478 kidney tumors have been surgically treated at our institution. We report the frequency and characteristics of OR in our patients, compared with renal cell carcinomas (RCC). We try to find out the rate of multifocality, bilateralism and other tumor association, and the number of neoplasms originally diagnosed as OR before surgery. Mean and median follow up: 36.86 and 13 months (1-193). Specific survival rate $100 \%$.

Results: We found 24 OR in 10 men and 12 women with a mean age of 59 years (34-84). 12 in the left kidney and 12 in the right one, one patient presenting oncocytomatosis. Tumor mean size was $4.64 \mathrm{~cm}$ (1-12.5 cm). Tumors were discovered incidentally in 17 cases. Presentation symptoms in the rest of patients were gross hematuria.

Conclusions: The rate of OR found in our sample population of renal tumors undergoing surgery matches other series already published. Two synchronic OR, but not metacronous, bilateral or metastatic tumors were found. All cases presented a benign evolution. 
$\mathrm{E}^{1}$ oncocitoma renal (OR) es un tumor benigno compuesto exclusivamente por células bien diferenciadas, de citoplasma eosinófilo con abundantes mitocondrias, denominadas "oncocitos". Este término fue acuñado por Hamperl en 1931. Jaffé en 1932 creó el término onkocytoma al describir un tumor de Warthin parotideo ${ }^{1}$, y en 1942 Zippel describió el primer $\mathrm{OR}^{2}$. Esta patología fue establecida como entidad independiente por Klein en $1976^{3}$.

El OR está considerado actualmente como tumor renal epitelial benigno, y se ha descrito su posible bilateralidad, multifocalidad y asociación a otros tumores renales tanto benignos como malignos. Aunque hay descritos algunos casos en que han mostrado un comportamiento maligno, al ser capaces de originar metástasis, actualmente se cree que estos casos fueron carcinomas de células renales (CCR), fundamentalmente del tipo cromófobo, incorrectamente diagnosticados.

Nos proponemos analizar en este trabajo la proporción de OR hallados en nuestra serie de tumores renales intervenidos, analizando sus características anatomopatológicas y comportamiento clínico, comparándolas con las presentadas por los CCR. Observamos su posible bilateralidad, multifocalidad y asociación con otros tumores. Pretendemos además determinar hasta qué punto los métodos diagnósticos de imagen permitieron en nuestra serie sospechar la naturaleza tumoral benigna previamente a la intervención.

\section{MATERIAL Y MÉTODOS}

Entre Enero de 1986 y Abril de 2005 hemos intervenido en nuestro Servicio 428 tumores renales. Realizamos en esta serie una revisión retrospectiva de los OR diagnosticados, estudiando la proporción que suponen dentro del total de tumores. Analizamos también la edad y el sexo de los pacientes, tamaño, lateralidad del tumor, proporción de casos incidentales, sintomatología de presentación y supervivencia. Comparamos los resultados con los presentados por los $367 \mathrm{CCR}$ diagnosticados en ese periodo de tiempo. Para ello fueron incluidos en una base de datos, creada para este fin, en el programa Access (Microsoft) y analizados mediante el paquete informático SPSS 11,5. Comparamos los resulta- dos de ambos grupos utilizando el test de la $\chi^{2}$. La estimación de las distribuciones de supervivencia fue calculada mediante el método de Kaplan-Meier, utilizando el test de Log Rank para compararlas.

La mediana y media del seguimiento fueron 13 y 36,86 meses respectivamente (1-193).

De los 22 casos diagnosticados se tuvo acceso para la revisión histopatológica a 15. Los hallazgos macroscópicos fueron recuperados del informe anatomopatológico de la pieza quirúrgica. Un único patólogo evaluó en las piezas recuperadas las siguientes características macroscópicas: carácter focal o multifocal, color del tumor, presencia o no de cicatriz central y áreas hemorrágicas. Entre las características microscópicas evaluadas destacamos: patrón arquitectural predominante, tipo de célula, grado de atipia celular, hipercromasia, presencia o no de nucleolo, actividad mitótica y formación de calcio.

Se realizó estudio inmunohistoquímico para citoqueratina pan y vimentina en aquellos cuatro casos en los que el diagnóstico planteaba dudas con otro tipo de neoplasia renal. Además en seis casos se tomaron muestras en glutaraldehido, durante el tallado de la pieza de resección, para estudio ultraestructural.

Respecto a los métodos diagnósticos, hacemos una revisión retrospectiva de los informes radiológicos de estos 22 pacientes, determinando en cuántos se hizo referencia a alguna característica que orientase hacia la benignidad tumoral, o incluso a su clasificación como OR.

\section{RESULTADOS}

Hemos encontrado 24 oncocitomas, en 10 hombres y 12 mujeres, lo que supone un 5,67\% de los pacientes intervenidos por neoplasia renal en esos años. La edad media ha sido 59 años (3484).

Los tumores se han distribuido con igual proporción en ambos riñones (12 en riñón izquierdo y 12 en derecho). En dos pacientes hemos encontrado multifocalidad. En uno de ellos, además de dos OR macroscópicos, de 1 y $3 \mathrm{~cm}$, se hallaron múltiples focos microscópicos de oncocitos (oncocitomatosis renal).

En ninguna de estas características hay diferencias significativas con los casos de CCR diag- 
nosticados, que presentan una edad media de 60.23 años (15-83) y asientan en el riñón izquierdo en el $44,5 \%$ de las ocasiones y en el derecho en el $55,5 \%$.

El tamaño tumoral medio de los OR ha sido $4,64 \mathrm{~cm}(1-12,5)$. Los CCR han resultado significativamente mayores al diagnóstico. Un 47,3\% de ellos fueron mayores de $7 \mathrm{~cm}$ (T2) en ese momento, frente a un $31,8 \%$ de los oncocitomas.

En 17 pacientes $(77,27 \%)$ el diagnóstico ha sido incidental, durante el estudio por patología urológica no relacionada con el tumor en 5 ocasiones, digestiva en 6, y ginecológica en 2. En el resto de pacientes el diagnostico incidental se realizó en estudio por hipertensión, síndrome anémico, estudio vascular por aneurisma aórtico y como planificación para cirugia por obesidad mórbida.

En los pacientes con clínica atribuible al tumor $^{5}$, ésta fue en todas las ocasiones hematuria.

En este apartado hemos encontrado diferencias estadísticamente significativas respecto a los CCR. En ellos, aunque la forma de diagnóstico más frecuente también es la incidental, ésta sólo supone un $47,1 \%$, siendo el estudio de síntomas urológicos no relacionados con el tumor y los estudios digestivos $(42,2 \%$ y $24,8 \%)$ los motivos de diagnóstico más frecuentes. En los CCR no incidentales, la hematuria $(55,3 \%)$ ha sido igualmente el síntoma inicial más frecuente.

Los principales hallazgos macroscópicos se recogen en la Tabla 1. Todos los tumores fueron descritos como masas esféricas, bien delimitadas y que comprimian el parénquima renal adyacente. Todos los casos estudiados eran de presentación unifocal excepto dos. La coloración fue descrita como marrón oscuro en 6 casos y amarillenta en 9. Tres casos presentaban áreas de fibrosis central y en siete casos se apreció áreas de hemorragia. En dos casos el riñón mostraba poliquistosis renal, en otros dos se asociaba a adenomas papilares. En uno se observó oncocitosis concomitante.

Los hallazgos microscópicos se resumen en la Tabla 2. Prácticamente la totalidad de los tumores estudiados mostraban un patrón arquitectural mixto, siendo el más común el acinar, donde las células tumorales se agrupan formando nidos compactos o acinos separados entre sí un estroma
Tabla 1

\begin{tabular}{lc}
\hline Unifocal/Mutifocal & $13 / 2$ \\
Amarillo pálido/marrón & $9 / 6$ \\
Cicatriz central & $3(20 \%)$ \\
Hemorragia & $7(46.67 \%)$ \\
Patología concomitante: & 5 \\
- Oncocitosis & 1 \\
- Poliquistosis renal & 2 \\
- Adenoma papilar & 2 \\
\hline
\end{tabular}

\section{Tabla 2}

\begin{tabular}{lc}
\hline Patrón arquitectural & \\
- Acinar & $12 / 15(80 \%)$ \\
- Tubular & $5 / 15(33,3 \%)$ \\
- Sólido & $8 / 15(53,33 \%)$ \\
- Pseudopapilar & $1(6,67 \%)$ \\
- Trabecular & 0 \\
Tipo de célula & \\
$\quad$ - Típica oncocítica & $15 / 15(100 \%)$ \\
$\quad$ - Picnosis & $12 / 15(80 \%)$ \\
- Célula clara & 0 \\
Atipia celular & $6 / 15(40 \%)$ \\
- Marcada & $2 / 15(14,2 \%)$ \\
Nucleolo & $13 / 15(86.7 \%)$ \\
Mitosis & 0 \\
Calcificación & $4 / 15$ \\
Necrosis & $(26.67 \%)$ \\
\hline
\end{tabular}

hipocelular. En cinco casos se apreció un patrón tubular, constituido por túbulos delimitados por células oncocíticas. En ocho casos se evidenció un patrón de crecimiento sólido, consistente en masas constituidas por nidos de células compactas separadas por finos capilares. En un solo caso existían áreas en que las células tumorales se disponian adoptando formas pseudopapilares.

En ningún caso se observó invasión vascular ni del tejido adiposo perirrenal. Además de las típicas células oncocíticas, presentes en el 100\% de los casos estudiados, se encontraron otras células de citoplasma más eosinófilo y núcleo picnótico pequeño y oscuro en 12 casos (80\%). El significado biológico de estas células es desconocido aunque se piensa que constituyen una variedad del oncocito clásico.

En 13 casos $(86,7 \%)$ el nucleolo era pequeño pero evidente. En el resto no se apreciaba.

Atipia nuclear fue encontrada en ocho casos, siendo en dos de ellos de carácter marcado, con- 
siderando como tal la presencia de núcleos de contornos irregulares, algunos polilobulados, aumentados de tamaño e hipercromáticos.

No se identificaron figuras de mitosis ni áreas de necrosis.

Calcificaciones distróficas fueron evidentes en 4 casos $(26,67 \%)$, sin observar áreas de metaplasia ósea.

El estudio inmunohistoquímico mostró positividad para citoqueratinas y negatividad para vimentina en los casos realizados.

En el estudio ultraestructural, los oncocitos mostraban un citoplasma repleto de mitocondrias y con escasez de otras organelas. Las mitocondrias tendian a ser uniformes y predominantemente redondeadas, con crestas lamelares dispuestas en paralelo. El núcleo era redondeado pudiendo apreciarse en muchos de ellos nucleolo.

Para llegar al diagnóstico a 21 de los pacientes se les realizó ecografía, a todos ellos se les hizo tomografía axial computerizada (TAC) y en dos casos el estudio se completó con una resonancia magnética nuclear (RMN). En dos casos los hallazgos tomográficos orientaron hacia un oncocitoma al encontrar la clásica imagen de cicatriz central. En otros tres orientaban a que pudiera tratarse de un tumor renal benigno ante la homogeneidad lesional, pero como diagnóstico menos probable tras el CCR. En dos de estos se solicitó RMN, no aclarando el diagnóstico en ninguno.

Sólo uno de los pacientes falleció, un año y medio después de la cirugía, por rotura de aneurisma aórtico. La supervivencia específica a los cinco años fue por tanto del $100 \%$, frente a un $81.30 \%$ en los CCR (Log Rank 0,1012) (Fig. 1).

No hemos encontrado ningún caso de metacronicidad, asociación a CCR, ni bilateralidad.

\section{DISCUSION}

Son pocas las series de OR publicadas en la literatura española, al no tratarse de una patología especialmente frecuente. Destacan la de 14 casos publicados por Monge et al. en $1994^{4}$, además de la revisión de los 45 casos publicados hasta 1992 realizada por Ballesteros et $\mathrm{al}^{5}$. En las demás ocasiones se publican de manera aislada o en series muy cortas. En la literatura

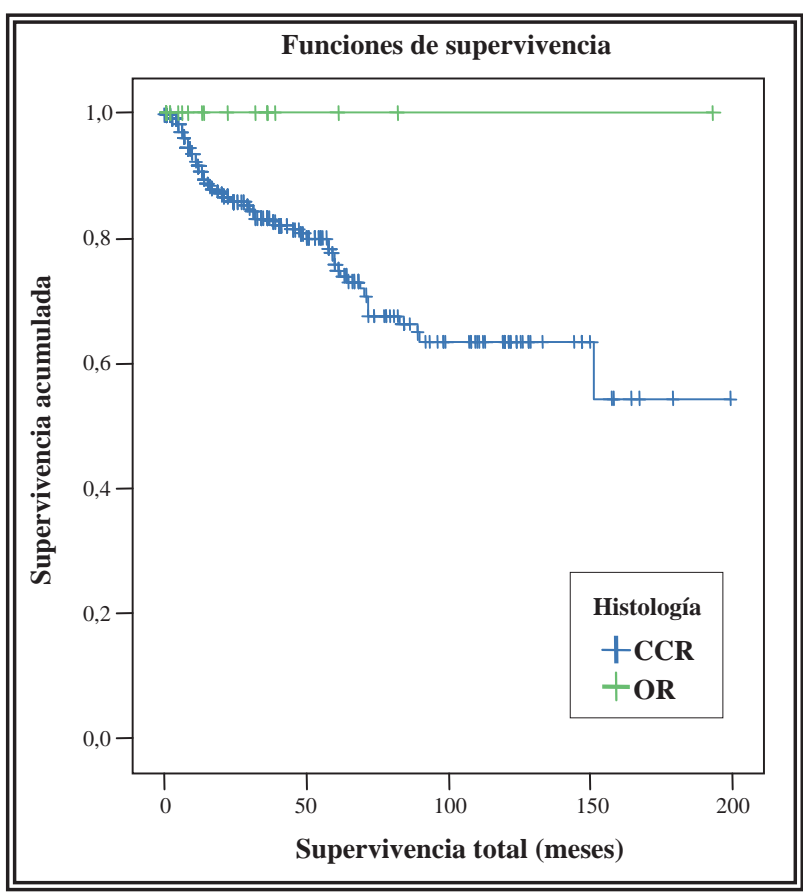

Figura 1: Curva Kaplan Meier comparando la supervivencia de OR y CCR.

anglosajona destacan la publicada en 1999 por Dechet et al, en la clínica Mayo, con 138 pacientes $^{6}$, y la serie de 70 casos por Pérez Ordóñez et al. en el Memorial Cancer Center de New York ${ }^{7}, \mathrm{y}$ la más reciente, de Gudbjartsson et al. con $45^{8}$.

$\mathrm{Su}$ incidencia es de aproximadamente un 3$10 \%$ de los tumores renales ${ }^{9-11}$, aunque se estima que alcanza hasta el $18 \%$ de los menores de cuatro $\mathrm{cm}^{6}$. Para algunos es por tanto el tumor renal benigno sólido más frecuente. Aunque el porcentaje de oncocitomas en nuestra serie se corresponde con estas frecuencias, no es el tumor sólido benigno intervenido más frecuente, habiéndose encontrado 25 angiomiolipomas. En la distribución respecto a sexos parece existir un predominio masculino $2-3 / 1^{6,10}$, aunque esto, como ocurre en nuestros casos, no es encontrado por todos los autores.

La edad media de presentación suele estar en torno a los 60 años ${ }^{4,10,11}$. Actualmente la forma de diagnóstico más frecuente es la incidental en el curso del estudio de otras patologías. Así sucede en nuestros pacientes, al igual que en los tumores renales considerados en su conjunto. De ser sintomáticos suelen mostrar, con una proporción distinta entre los distintos trabajos: dolor lumbar, sintomas constitucionales, sindrome de 
Wunderlich, masa en flanco, hipertensión, alteraciones de la función renal y hematuria ${ }^{12}$. Esta última fue la forma de presentación única en nuestra muestra.

No parece haber preferencias respecto a la lateralidad del tumor, y su tamaño medio oscila entre de 3,2 a $8,7 \mathrm{~cm}^{4,9,11,13}$.

La mayoría nuestros casos se corresponden con la definición macroscópica típica de los oncocitomas. Suelen ser tumores esféricos, pseudoencapsulados y bien delimitados que simulan la coloración del parénquima renal adyacente, aunque frecuentemente adquieren una coloración marrón oscura debido a su gran contenido en sangre. En nuestro grupo 6 casos (40\%) mostraron este color mientras que el resto se describieron como amarillo pálido. Un hallazgo descrito clásicamente como característico es la presencia de una cicatriz central, apareciendo en el 33-54\% de los oncocitomas según las series más recientes $^{10}$. En nuestra serie lo hemos encontrado en tres casos. Este hecho no se puede considerar diagnóstico ya que se observan en gran cantidad neoplasias renales. Aunque la hemorragia intratumoral es rara, fue macroscópicamente evidente en 7 casos (46,67\%) (Fig. 2).

Con cierta frecuencia se pueden encontrar hallazgos más atípicos, no invalidando el diagnóstico de OR y por tanto su previsible comportamiento benigno. Estos son la invasión de grasa perinefrítica, necrosis focal e invasión microvascular. En la serie publicada por Amin et al., los dos primeros aspectos estuvieron presentes en un $11,3 \%$ y un $17,4 \%$ respectivamente, manteniéndose la supervivencia a los 5 años en un $100 \%{ }^{12}$. En nuestro grupo no se han encontrado afectación del tejido adiposo perirrenal, necrosis, ni invasión microvascular en ninguno de los casos estudiados.

Microscópicamente los OR están constituidos células redondas o poligonales de citoplasma eosinófilo amplio, granular y de bordes bien definidos, con núcleo generalmente único y central, de cromatina finamente granular, aunque no es raro observar formas binucleadas. El nucleolo suele ser pequeño pero evidente. Las células tumorales se disponen formando nidos sólidos, acinos o túbulos sobre un estroma fibroso e hialino. El patrón sólido consiste en acinos o grupos

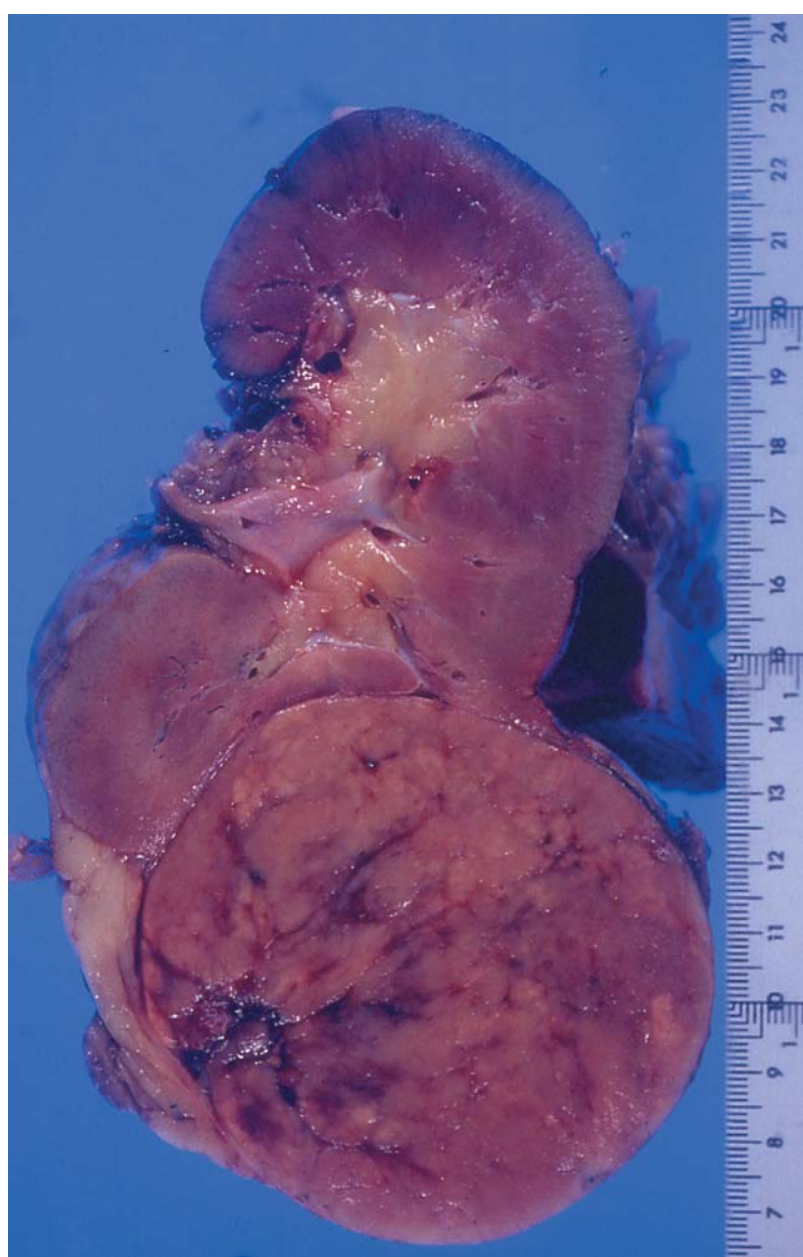

Figura 2. Aspecto macroscópico del OR. Tumor pardo, bien circunscrito, no encapsulado, con pequeños focos de hemorragia intratumoral.

compactos de oncocitos separados entre sí por finas estructuras capilares, en ausencia de estroma hipocelular. En nuestra serie la mayoría de los tumores presentaban un patrón arquitectural mixto donde el patrón predominante fue el acinar (Fig. 3), acompañándose de áreas tubulares en cinco casos y sólidas en ocho (Fig. 4) ${ }^{14}$. Únicamente en un caso se observó patrón pseudopapilar, no siendo el dominante, pues de lo contrario no podría hacerse el diagnóstico de oncocitoma ${ }^{12}$.

El resto de los parámetros citológicos estudiados, y presentes en nuestro estudio (Tabla 2) han sido ya descritos por otros autores en este tipo de tumores. Algunos, como la presencia o no de nucleolo y la atípia celular han sido utilizados como parámetros en un intento de gradación de los oncocitomas. Sin embargo ninguna de las características mencionadas antes parece tener 


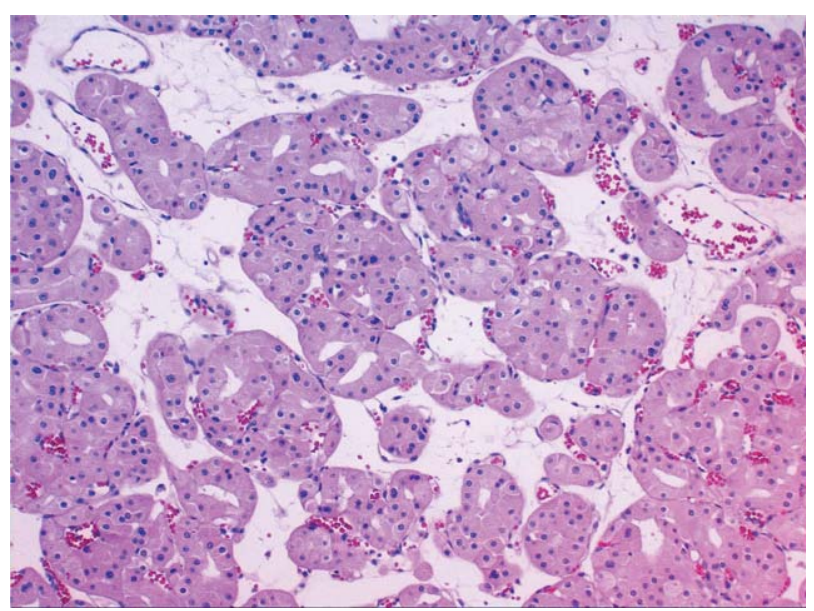

Figura 3: Patrón organoide o clásico, con células eosinófilas formando nidos.

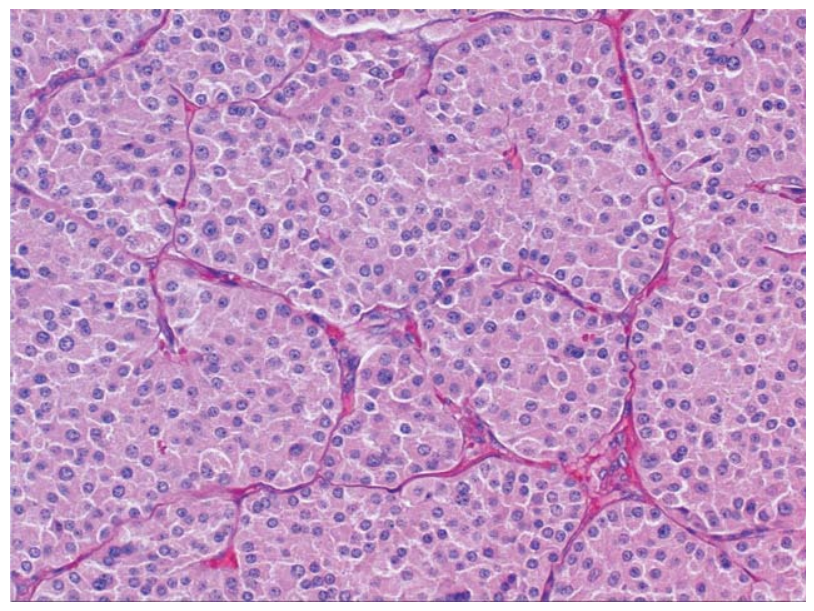

Figura 4: Patrón sólido.

relevancia clínica ni valor pronóstico. No se contempla en el diagnóstico la presencia de mitosis abundantes o atípicas ${ }^{15}$.

Ultraestructuralmente destaca la distribución difusa de mitocondrias redondeadas, con escasez de otros organelos, además de pequeños acúmulos de microvesículas (Fig.5). Este último hallazgo, además de que en ocasiones expresan inmunorreactividad especifica para la anhidrasa carbónica $\mathrm{C}^{12,16}$, ha puesto luz en el discutido origen de estos tumores. Actualmente parece aceptado que proceden de las células intercalares de los túbulos colectores distales, que presentan habitualmente este tipo de microvesículas ${ }^{15}$. Este origen es común con el carcinoma renal de células cromófobas, lo que explica la dificultad que en ocasiones supone hacer diagnóstico diferencial

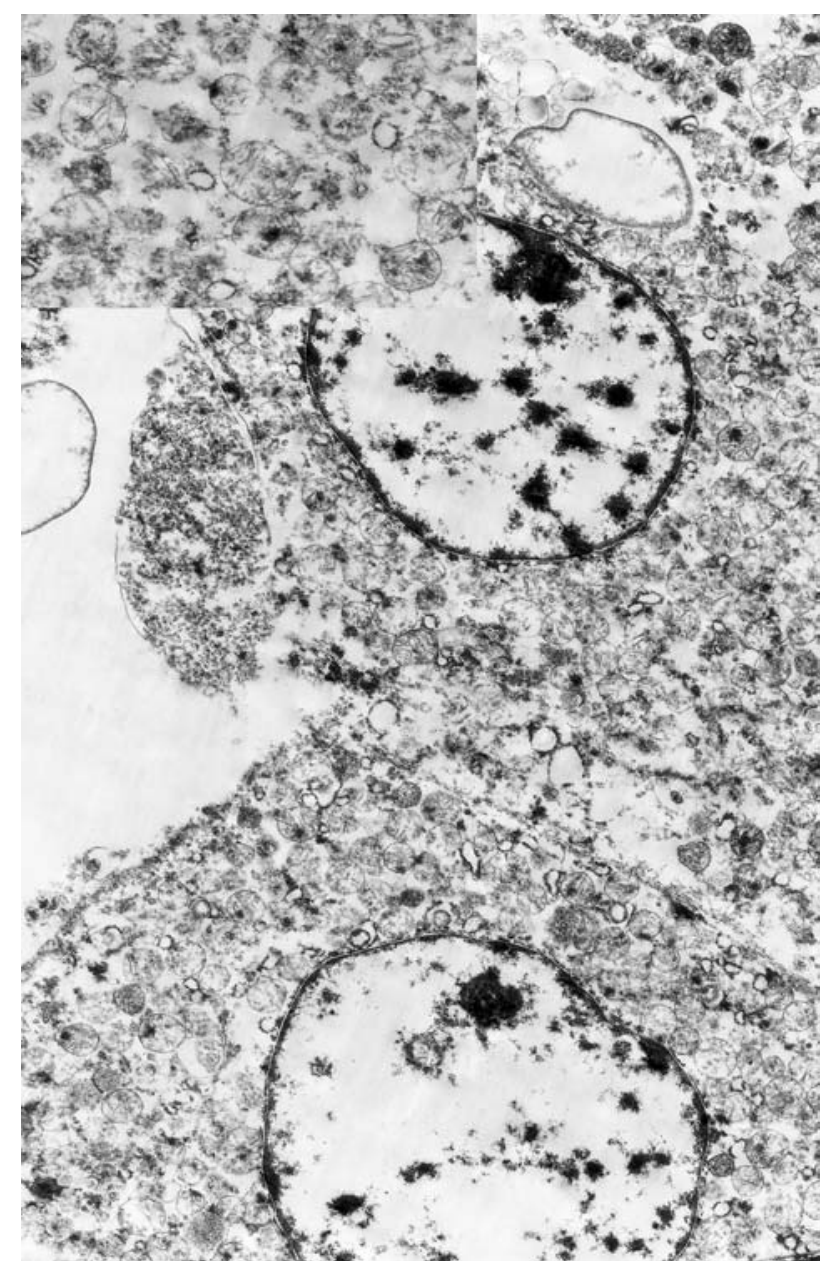

Figura 5. Microscopía electrónica: núcleos redondeados, con cromatina dispersa homogéneamente. En el detalle del recuadro observamos abundantes corpúsculos redondeados, correspondientes a mitocondrias.

entre ambos ${ }^{16} \mathrm{~A}$ esta dificultad para el diagnostico diferencial entre determinados tipos de RCC se le atribuyen los casos de OR agresivos, incluso metastásicos, que podemos encontrar en la literatura. Para Amin et al., la inclusión de tumores con carcinoma de células claras sincrónico, la inadecuada toma de muestras y la falta de criterios estandarizados han contribuido a esta confusión ${ }^{12}$.

Aunque es un tumor habitualmente único, la multifocalidad, bilateralidad y metacronicidad ha sido frecuentemente descrita ${ }^{17,18}$. La frecuencia de multifocalidad oscila desde el 2,5 al $16 \%{ }^{6,10}$, coincidente con el 9,09\% encontrado en nuestra serie. La bilateralidad, estimada en las publicaciones en torno al 5\%, no ha estado presente en nuestros casos. Tampoco hemos detectado lesiones metacrónicas, en un seguimiento medio de 
36,86 meses (1-193). En la amplia serie de la clínica Mayo encontraron que ésta se presentó en un 4\% de sus pacientes, una media de 9.5 años después del diagnóstico inicial. Es posible por tanto que con un seguimiento mayor detectemos algún caso. No obstante los autores de este artículo mencionan la posibilidad de que en ocasiones, cuando se ha practicado cirugía ahorradora de nefronas, lo identificado como tumor metacrónico se trate en realidad de una lesión sincrónica no identificada en la cirugía inicial. No obstante insisten en tener en cuenta la posibilidad de metacronicidad años después de la cirugía inicial, por lo que sugieren realizar cirugía conservadora de nefronas cuando sea posible.

Se han publicado también casos de asociación a otros tumores renales benignos ${ }^{19}$ o malignos ${ }^{20,21}$, tanto de una manera sincrónica o metacrónica. En nuestros pacientes hemos encontrado dos casos asociados a adenomas corticales de características papilares, además de dos casos de multifocalidad, uno de ellos presentando además oncocitomatosis. Curiosamente estos dos últimos enfermos se encontraban en hemodiálisis por insuficiencia renal crónica terminal. Esta es una entidad descrita en 1982 por Warfel and Eble ${ }^{22}$, que encontraron más de doscientos focos de oncocitoma en un paciente, de manera bilateral. Desde entonces se han descrito muy escasos casos similares, alguno de ellos de forma unilateral y otros, al igual que en el nuestro, en pacientes sometidos a diálisis durante largo tiempo $23-25$. Para algunos autores esta entidad podría entenderse como el paso siguiente a una hiperplasia oncocítica nodular multifocal de asiento renal. Esto ya ha sido sugerido en los oncocitomas de glándulas salivares y explicaría la posibilidad de desarrollo de oncocitomas múltiples de forma simultánea o metacrónica ${ }^{18}$.

Aunque en nuestro estudio no hemos encontrado asociación entre OR y CCR, distintos autores la han estimado entre un 2 y un $32 \% 6,11,15$. Esto sugiere que aunque dichas neoplasias se consideran actualmente entidades independientes, deben tener cierta relación, apoyada por la presencia de CCR con hallazgos oncocíticos y por el hallazgo ocasional de CCR "enterrados" en el seno de un OR.
La secuencia diagnóstica de los OR es la empleada en los tumores renales en general. La ecografía se ha erigido en la prueba con la que más frecuentemente se diagnostica inicialmente la presencia de una masa renal sólida. Tras ella suele solicitarse una TAC, que suele mostrar al OR como una lesión sólida con buena demarcación y homogénea, de baja atenuación (menor que el parénquima circundante). No obstante la presencia de necrosis o focos hemorrágicos pueden producir atenuación variable. En algunos de ellos se observa una zona de baja atenuación central que se corresponde con la zona de cicatriz estrellada (Fig. 6). Otros criterios orientativos para el diagnóstico de oncocitoma son realce hipodenso tras contraste intravenoso, patrón vascular en radios de rueda y ausencia de margen redondeado. No obstante, estos hallazgos no son patognomónicos, por lo que el diagnóstico diferencial es complejo, existiendo trabajos de revisión retrospectiva de TAC en los que sólo el $12,2 \%$ de OR se diagnosticaron correctamente ${ }^{26}$. En nuestra serie este valor es de 9,9\% (2/22) si hablamos de sospecha de OR, y de 22,73\% (5/22) si lo hacemos de tumor benigno. Además en RCC menores de $3 \mathrm{~cm}$ la posibilidad de error es mayor en los tumores de menor tamaño, encontrándose en la serie de Davidson que hasta un 42\% de CCR menores de $3 \mathrm{~cm}$ se diagnosticaron como OR usando los criterios de homogeneidad o presencia de cicatriz central ${ }^{27}$.

$\mathrm{El}$ aspecto en RMN en los OR no está muy establecido, dada la baja incidencia de esta neo-

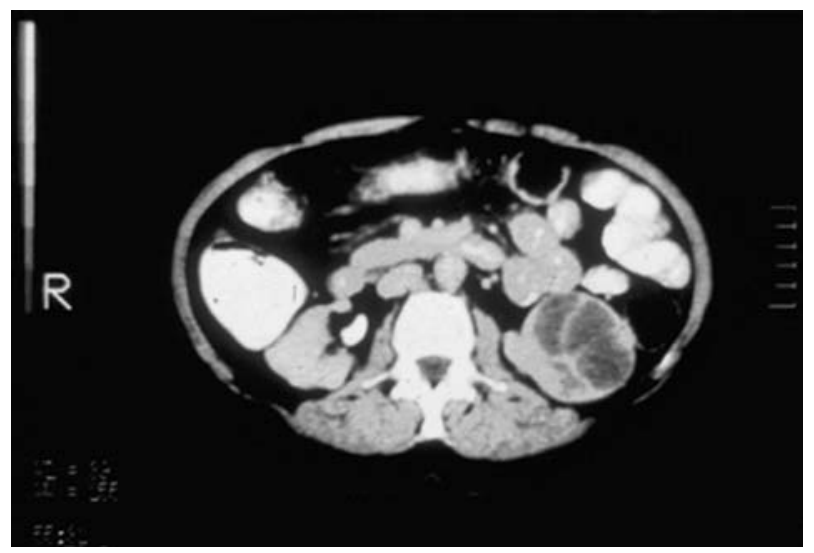

Figura 6. Imagen de TAC abdominal en la que se aprecia tumoración renal izquierda con imagen de cicatriz central, compatible con oncocitoma. 
plasia y la necesidad de hacer RMN en todas las masas renales sólidas para encontrarlas. Parece que suelen presentarse una disminución de la señal de forma homogénea en $\mathrm{T} 1$ y con incremento de la intensidad de la señal en T2. Se apunta la utilidad de esta prueba diagnóstica para diferenciar si una lesión hipodensa central en una TAC es debido a necrosis tumoral o fibrosis $^{13}$.

\section{CONCLUSIONES}

El OR es un tumor de evolución benigna. Sus especiales características de metacronicidad, posible bilateralidad y asociación a CCR hacen recomendable un seguimiento continuado.

En nuestra serie la frecuencia de OR concuerda con la encontrada por otros autores. No ocurre así respecto a la metacronicidad y la asociación con CCR. No obstante es probable que con un seguimiento medio mas prolongado podamos encontrar estas asociaciones, según se desprende de la literatura.

Dado el difícil diagnóstico prequirúrgico de esta neoplasia, el manejo es en general común con el del resto de neoplasias sólidas de riñón.

\section{REFERENCIAS}

1. Jaffe RH. Adenolymphoma of the parotid gland. Am J Cancer. 1932;16:1415.

2. ZippeL L. Zur kenntnis der onkocyten. Virchow Arch Path Anat. 1942;308:360-382.

3. Klein MJ, Valensi QJ. Proximal tubular adenomas of kidney with so called oncocytic features. A clinicopathologic study of 13 cases of a rarely reported neoplasm. Cancer. 1976;38(2): 906-914.

4. Monge Miralles JM, Gutiérrez Baños JL, Martín García B, Hernández Rodríguez R, Portillo Martín JA, Correas Gómez MA, et al. Oncocitoma renal: presentación de 14 casos y revisión de la literatura. Arch Esp Urol. 1994;47(6):564-570.

5. Ballesteros JJ, Pérez Herms S, Cortadellas R, Guzmán A, Castellanos R, Munne A. Revisión de 45 casos de oncocitomas renales publicados en nuestro pais y aportación de 2 nuevos casos. Actas Urol Esp. 1992;16(2):113-119.

6. Dechet CB, Bostwick DG, Blute ML, Bryant SC, Zincke H. Renal Oncocytoma: multifocality, bilateralism, metachronous tumor development and coexistent renal cell carcinoma. J Urol. 1999;162(1):40-42.

7. Pérez-Ordonez B, Hamed G, Campbell S, Erlandson R, Russo P, Gaudin P, et al. Renal Oncocitoma: A Clinicopathologic Study of 70 cases. Am J Surg Pathol. 1997;21(8): 871-883.

8. Gudbjartsson T, Hardarson S, Petursdottir V, Thoroddsen A, Magnusson J, Einarsson GV. BJU Int. 2005;96(9):12751279.

9. Requena MJ, López Beltrán A, Anglada FJ. Neoplasias renales. Diagnóstico y tratamiento. P35-41. Ed Servicio de Urologia. Hospital Universitario Reina Sofia. 2001.
10. Schatz SM, Lieber MM. Actualización sobre el Oncocitoma. Current Urology Reports (ed española) 2003;2(4):187-193.

11. Romis L, Cindolo L, Patard JJ, Messina G, Altieri V, Salomon L, et al. Frequency, clinical presentation and evolution of renal oncocytomas: multicentric experience from a european database. Eur Urol. 2004;45(1):53-57.

12. Amin MB, Crotty TB, Tickoo SK, Farrow GM. Renal Oncocitoma: a reappraisal of morphologic features with clinicopathologic findings in 80 cases. Am J Surg Pathol. 1997;21:1-12.

13. Herranz Amo F, Verdu F, Castaño I. Oncocitoma renal. En: Herranz Amo F. Tumores de Riñón. Ed Doyma. 2001;245251.

14. Renshaw AA. Subclasification of renal cell neoplasms: an update for the practising pathologist. Histopathology. 2002; 41:283-300.

15. Chao DH, Zisman A, Pantuck AJ, Freedland SJ, Said JW, Belldegrun AS. Changing concepts in the management of renal oncocytoma. Urology. 2002;59:635-642.

16. Akhtar M. Renal oncocytoma versus chromophobe renal cell carcinoma: debate goes on. Adv Anat Pathol. 2005; 12(3): 152-153.

17. Licht MR, Novick AC, Tubbs RR, Klein EA, Levin HS, Streem SB. Renal oncocytoma: clinical and biological correlates. J Urol. 1993;150:1380-1383.

18. Sánchez-Chapado M, Angulo Cuesta J, Rodríguez de Bethencourt Codes F, Ontoria J, Prieto Chaparro C, et al. Oncocitoma renal bilateral sincrónico. Arch Esp Urol. 1995; 48(9):909-913.

19. Pillay K, Lazarus J, Wainwright HC. Association of angiomyolipoma and oncocytoma of the kidney: a case report and review of the literature. J Clin Pathol 2003;56:544547.

20. Walsh CA, Guinlan DM. Oncocytoma and synchronous urothelial carcinoma in same kidney: previously unreported association. Urology 2005;66(1): 194

21. Pachón Sánchez D, Palou Redorta J, Rosales Bordes A, Navarro Genta M, Villavicencio Mavrich H. Carcinoma de celulas renales asociado a oncocitoma renal contralateral metacrónico. Arch Esp Urol 2005; 58(2):175-178.

22. Warfel KA, Eble JN. Renal oncocytomatosis. J Urol. 1982; 127:1179-1180.

23. Shiga Y, Suzuki K, Tsutsumi M, Ishikawa S, Gotoh M, Shimokama, et al. Renal oncocytomatosis in a long-term hemodialysis patient treated by laparoscopic surgery. Int $\mathrm{J}$ Urol. 2002; 9(11): 646-649.

24. AcH S, Chapuis H, Mottet N. Metachronous Renal Oncocytomatosis. Urol Int. 2003;70(3):241-243.

25. Farkas LM, Szekely JG, Karatson A. Bilateral, multifocal renal oncocytomatosis with rapid progression leading to renal insuffiency. Nephrol Dial Transplant 1999;14(9): 2262-2263.

26. Wildberger JE, Adam G, Boeckmann W. Computed tomographt characterization of renal cell tumors in correlation with histopathology. Invest Radiol 1997;32:596-601.

27. Davidson AJ, Hayes WS, Hartman DS, McCarthy WF, Davis CJ. Renal oncocytoma and carcinoma:failure of differentiation with CT. Radiology 1993;186:693-696.

Dr. E. Argüelles Salido

earguelles@telefonica.net

(Trabajo recibido el 10 de noviembre 2005) 